\title{
14
}

\section{Expert design knowledge: a case study in dental education}

\author{
J. C. Davenport \\ F. J. Fitzpatrick \\ D. A Randell
}

University of Birmingham

United Kingdom
P. Hammond

Brunel University

United Kingdom

M. G. de Mattos

Sao Paulo University

Brazil

\begin{abstract}
The expertise of teachers of prosthetic dentistry in partial denture design (the design of removable partial denture, a type of dental prosthesis) has been elicited by identifying a collection of design rules and sampling expert reaction to them by first surveying 10 experts individually and then the prosthetic departments in all 17 dental schools in the British Isles. The surveys revealed an unsuspected consensus concerning the rules. This design expertise will be disseminated through RaPiD, a knowledge based assistant for the design of partial dentures. It is expected that by incorporating assistant rules into this design, which have been shown to be widely supported, the acceptability and usefulness of $\mathrm{RaPiD}$ for clinical education will be increased. In order to facilitate the incorporation of these rules into $\mathrm{RaPiD}$ semi-automated translation, rather than hand coding as PROLOG programs, is being investigated.
\end{abstract}

Main conference themes: artificial intelligence, tutoring

Educational areas: higher education

Study topics: life sciences/medicine

Secondary keywords: computer aided design, expert systems, knowledge based 


\section{INTRODUCTION}

It is more than ten years since the so-called knowledge acquisition bottleneck was first highlighted and there is now a substantial literature covering many aspects of knowledge elicitation and knowledge engineering [1]. The two aspects which we concentrate on here, the use of multiple experts and a domain involving expert design, are beginning to receive more focused attention [2, 3]. In this paper, we describe how we captured the design expertise of a group of more than 70 experts and how it is being encoded in RaPiD [4], a knowledge based assistant for designing a removable partial denture (RPD), a type of dental prosthesis.

The motivation for the production of RaPiD is the well recognized, world wide and large scale delegation by dentists of the responsibility for the design of RPDs to technicians who do not have the necessary clinical information or training to undertake this task [5]. The steadily increasing computerization of dental schools, postgraduate centres and dental practices has created the opportunity to introduce computer support to help solve this problem. The $\mathrm{RaPiD}$ project which is directed at providing this support, has two aims:

1. to produce an effective educational tool for both undergraduate and postgraduate education in this design domain, an educational aspect which will be strengthened by the future inclusion of context sensitive computer assisted tutorials [6];

2. to enhance a dentist's clinical expertise with expert design knowledge so that effective prosthesis design can be carried out in the surgery to the benefit of the patient.

\section{Rapid prototyping and development of RaPiD}

In the first stages of the project, we employed a single expert who had more than 25 years' experience in both the educational and clinical aspects of dental prosthesis design. His textbook on the subject [6] provided an additional and comprehensive source of design expertise for the knowledge engineer during the informal knowledge acquisition sessions. A preliminary version of RaPiD was rapidly prototyped in MacPROLOG on a Macintosh microcomputer to provide the expert with early insight into the educational potential of a design assistant and also to experiment with a graphical user interface. The interface and the functionality have retained much of the character of the original although $\mathrm{RaPiD}$ has been redesigned along more robust and logically sound lines [8]. The design of the RPD is now represented as a (logic) database of subcomponents with expert design rules acting as integrity constraints on 
updates to the database which result from a user's amendments of the developing design.

A CAD style interface with direct manipulation of icons and critiquing of incorrectly manipulated components has proved to be popular with users and is similar to the hand drawing of designs which is current practice. A design window is divided into a section containing the tool icons and a larger area containing an arch of teeth in which the prosthesis is designed (Fig. 1). When users violate a design rule a critiquing message notifies them of the fact (Fig. 2).

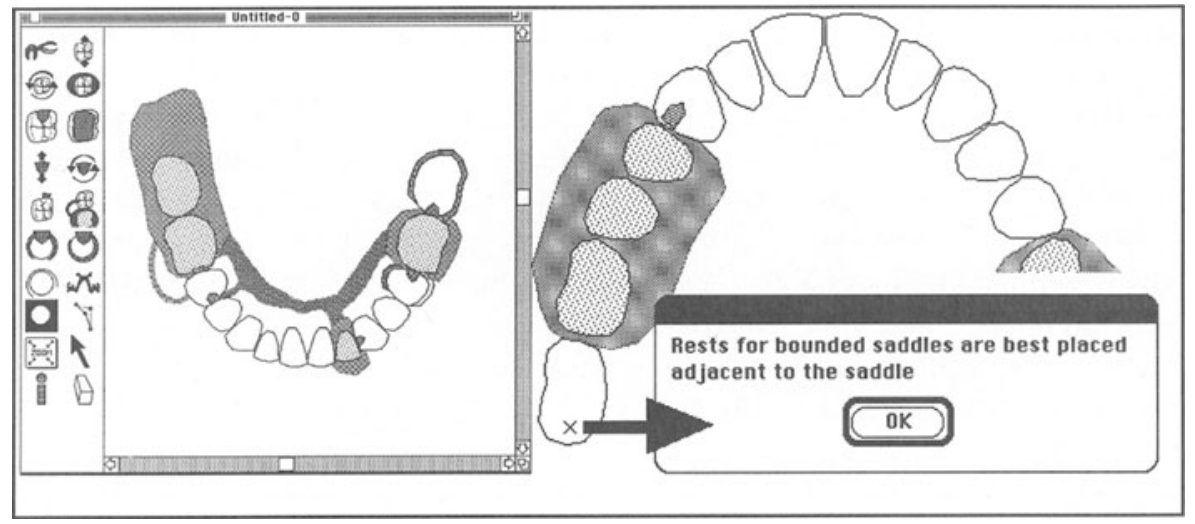

Fig. 1 A completed design produced in $\mathrm{RaPiD}$

Fig. 2 A critique of the user's design alteration

The dental expert and knowledge engineers have worked closely throughout the project so as to avoid known problems contributing to knowledge base systems project failure, such as incomplete knowledge acquisition and unsuitable interface design. Widespread exposure of the RaPiD prototype has taken place at dental workshops, conferences, through student use and through evaluation by dental practitioners. This has elicited favourable responses and has provided some constructive criticism for improvements, several of which have subsequently been incorporated into redesigned versions of RaPiD.

During the implementation of the prototype version of RaPiD and its early redevelopment the knowledge elicitation approach adopted was quite standard in its use of face to face interviewing. Inevitably, as the prototype developed, the expert became better informed and more demanding about the capabilities of a fully-fledged implementation. At the same time the knowledge engineers acted as apprentice dental prosthesis designers and acquired significant design expertise, a common side-effect of knowledge acquisition [9]. In addition, 
protocol analysis sessions were held in which the expert worked at real design problems while thinking aloud [10].

\section{Second phase of knowledge acquisition by survey of multiple experts}

While RaPiD was being re-implemented in accordance with the logic database model, the opportunity was taken to extend the source of expertise to a wide group of dental prosthetic teachers. This was primarily motivated by the desire to make the software as widely acceptable in dental education as possible. There are also additional advantages in involving multiple experts: groups often perform better than individuals in complex tasks; they can produce more and better solutions; they can clarify in discussion mutual understanding and even inspire group members to new insights [11].

The wider consultation began with a literature review undertaken by a prosthetic specialist. This review covered the RPD design literature in English of the last 10 years, also including the major textbooks and key papers from 1950 onwards. The outcome of this survey was a list of 119 design rules which formed the basis for a questionnaire aimed at identifying which rules received majority support. Diagrams, produced using RaPiD, were included to illustrate the design rules (Fig. 3). The rules were presented in such a way that the reader would be likely to provide a mixture of positive and negative responses.

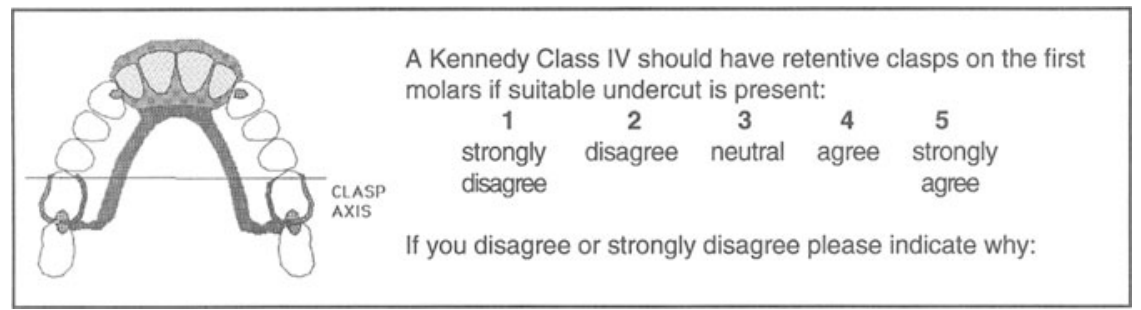

Fig. 3 A design rule in the survey questionnaire

\section{The Birmingham Survey}

Initially, the design rules questionnaire was piloted by circulating it to all ten dental prosthetic specialists at the School of Dentistry in Birmingham. This was to evaluate and refine the questionnaire and to obtain a measure of the variation in opinion within a group of experts. These local experts also suggested six more design rules to be added to the original 119. 


\section{The British and Irish dental school survey}

A copy of the revised questionnaire was sent to all of the departments of prosthetic dentistry in the other 16 dental schools in the British Isles with a request that it be completed to reflect the particular department's approach to design, rather than the view of any individual specialist. All of the schools replied. For each of the 125 rules the number of responses in each category (strongly agree to strongly disagree) was recorded and the total scores for each category were expressed as a percentage of the overall response (Fig. 4).

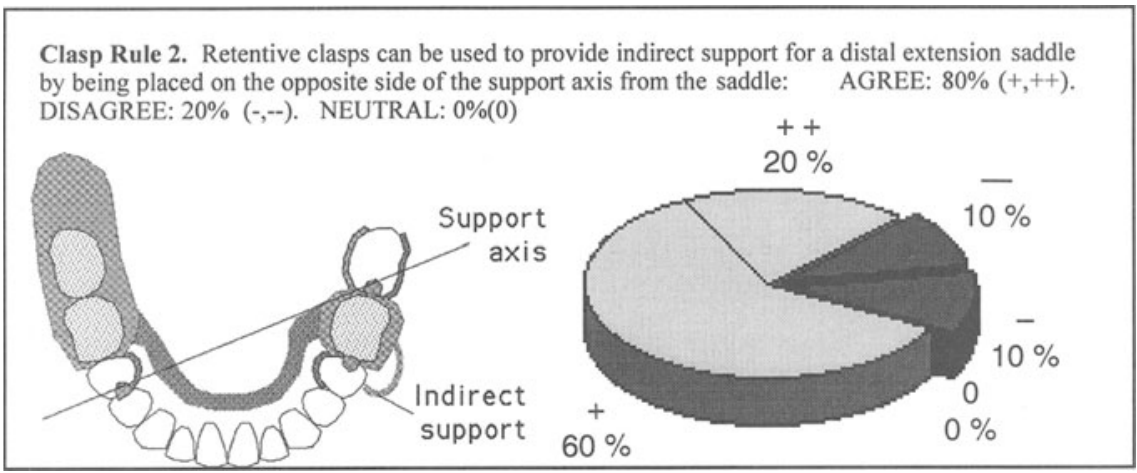

Fig. 4 Example of a design rule and the recorded level of agreement and disagreement

It was found that nine or more, i.e. in excess of 50 per cent, of the dental schools were in agreement on whether or not $111(89 \%)$ of the rules could be supported (Tables I and II).

Table 1 Overall level of agreement between schools

\begin{tabular}{lcc}
\hline & Number & Per cent \\
\hline Rules with which 9 or more schools were in agreement & 98 & 78.4 \\
Rules with which 9 or more schools were in disagreement & 13 & 10.4 \\
Rules with which 8 or fewer schools were in agreement or disagreement & 14 & 11.2 \\
TOTAL & 125 & 100 \\
\hline
\end{tabular}


Table 2 Range of agreement between schools

\begin{tabular}{lllllllllll}
\hline $\begin{array}{l}\text { Number of schools } \\
\text { in agreement }\end{array}$ & $<9$ & 9 & 10 & 11 & 12 & 13 & 14 & 15 & 16 & 17 \\
\hline Number of rules & 14 & 8 & 7 & 17 & 15 & 6 & 11 & 17 & 18 & 12 \\
& & & & & & & & & & \\
Percentage of rules & 11.2 & 6.4 & 5.6 & 13.6 & 12.0 & 4.8 & 8.8 & 13.6 & 14.4 & 9.6 \\
\hline
\end{tabular}

The results of the survey reflect the views of some 70 prosthetic specialists. The staff at the 17 schools comprise virtually all the recognized dental prosthetic teachers currently active in the British Isles. It is this group which provides both the undergraduate and postgraduate education in the speciality and therefore exerts the major influence on attitudes and practice of dental students and practitioners in the design of the type of prosthesis which RaPiD supports.

\section{International survey of design rules}

Some preliminary work on clarifying international viewpoints has been undertaken, as it is known that there are distinctive national characteristics in prosthesis design. This has identified a future requirement for some RaPiD users to have their own set of design rules supporting a local approach. However, it will be some time before we can provide software tools for individual users to include their own design rules in RaPiD. Such generic knowledge editing tools have received a good deal of attention recently [12] but activities such as graphical design in a clinical domain are much more difficult to tackle in this way.

\section{Implementing the acquired knowledge}

As the overall aim of the project is to produce a system which is acceptable to the majority of dental prosthetic teachers, rules which received support from at least 50 per cent of the dental schools were considered for inclusion. However some rules, especially those related to three-dimensional information, have had to be put aside for the moment until $\mathrm{RaPiD}$ is sufficiently developed to be able to cope with them.

Nineteen of the design rules about which there was a consensus, already formed part of the RaPiD knowledge base. Others required no further extension to the logic database model or the underlying ontology of the RPD design domain. The rules not yet implemented which are expected to cause the most difficulty in terms of knowledge representation, are likely to be those involving the most complex geometrical reasoning. For example, Clasp Rule 22 can be presented mathematically prior to inclusion in RaPiD (Fig. 5). 


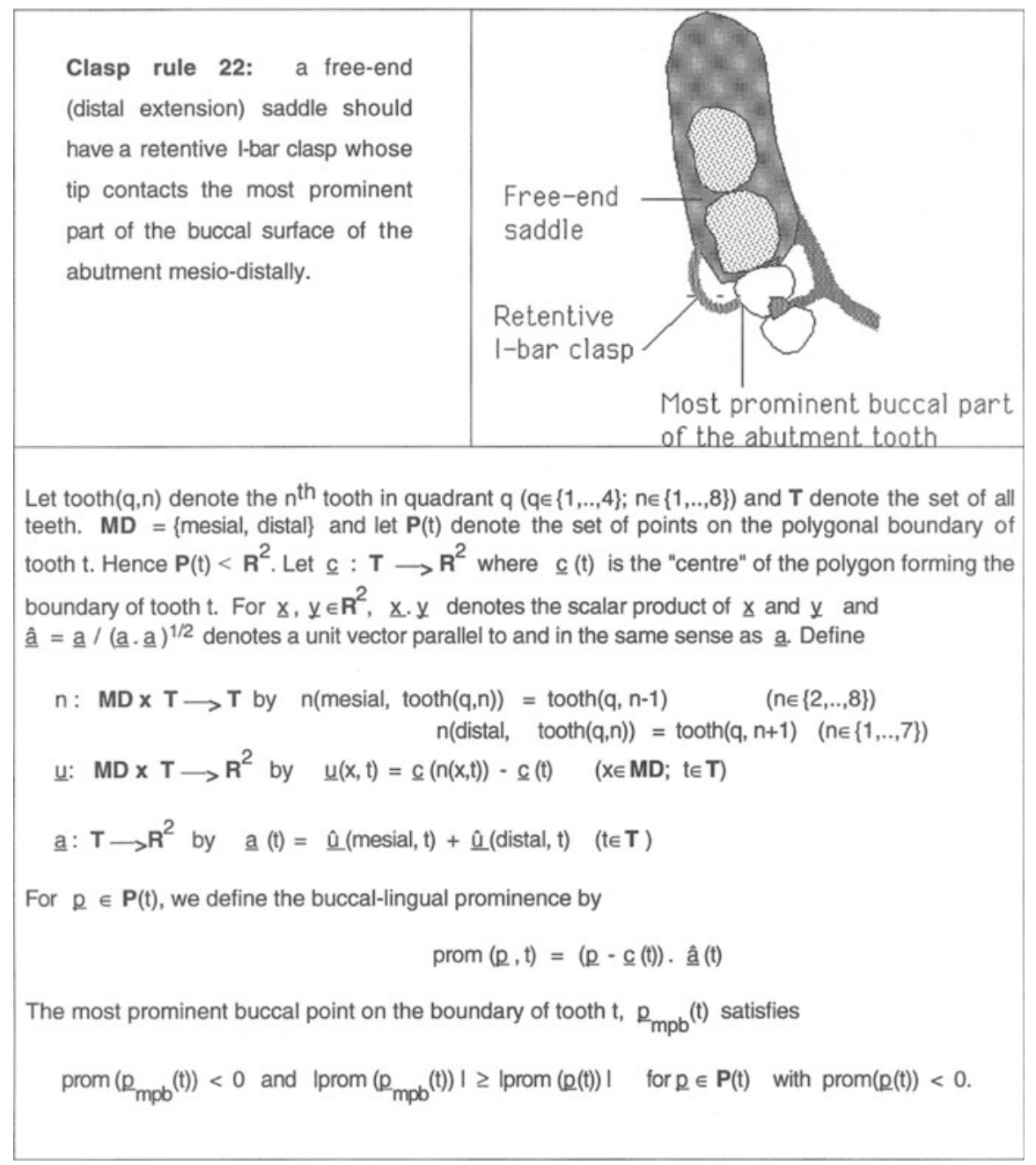

Fig. 5 Mathematical representation of Clasp Rule 22 prior to inclusion in RaPiD

These mathematical definitions can be viewed as semiformal specifications of the design expertise captured during the knowledge elicitation exercise. However, some of the rules are not so easy to represent in this fashion, as is demonstrated by the example in Fig. 6 which contains the rather vague term 'as far as possible'. 
Clasp rule 23: a unilateral free-end (distal extension) saddle denture (Kennedy II) should have one clasp as close to the saddle as possible and the other as far posteriorly as possible on the other side of the arch.

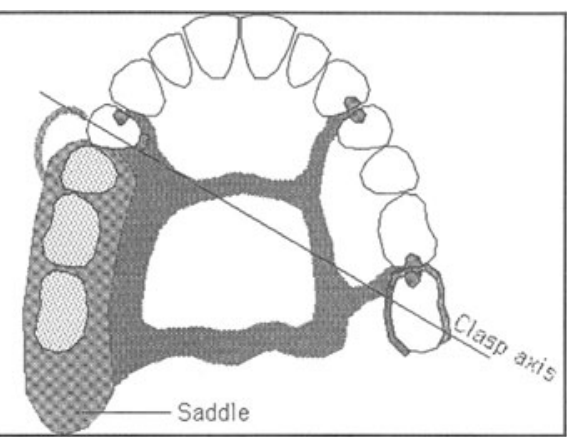

Fig. 6 Example of a design rule which is difficult to present mathematically

The survey of specialist teachers reported here has identified a large number of design rules. Rather than hand coding these as PROLOG programs we are investigating a semi-automated translation for improved speed and accuracy.

\section{CONCLUSIONS}

Recent studies of the methodology employed in knowledge acquisition have suggested that eliciting the expertise of many experts, as opposed to reliance on a single expert, is likely to improve the acceptability, accuracy, completeness and reliability of rules contained in a knowledge based system [2]. Our experience, gained from surveying specialists in prosthetic dentistry at all 17 schools of dentistry in the British Isles has, we believe, reinforced this view. In fact the survey has revealed a previously unsuspected consensus concerning rules for the design of removable partial dentures. Given the thorough nature of this survey we believe that the RPD design rules which have been identified as receiving majority support, can now be incorporated into the RaPiD system with confidence. However, there is a need to build versions of RaPiD to suit local requirements where these do not conform to the set of rules established as the norm.

In future, when the RaPiD software is more widely used in dental schools and hospitals, we will be able to build local, national and possibly international databases of symbolic representations of designs for subsequent analysis and semi-automated review. Such databases will allow thorough evaluation of the effectiveness of knowledge transfer by RaPiD. 


\section{ACKNOWLEDGEMENTS}

M.G. de Mattos has been supported under FAPESP (Fundaçãs de Ampaio à Pesquisa do Estads de São Paulo) while an academic visitor to the RaPiD project; F.J. Fitzpatrick and D.A. Randell were supported under the HEFCE Knowledge Based Systems Initiative.

\section{REFERENCES}

1. Neale, I.. M. (1988) First generation expert systems: a review of knowledge acquisition methodologies. The Knowledge Engineering Review 3 (2) pp. 105146.

2. Liou, Y. I. (1992) Collaborative knowledge acquisition. Expert Systems with Applications 5, pp. 1-13.

3. Shaw, M. L. G. (1989) A grid-based tool for knowledge acquisition: validation with multiple experts. KASI, pp. 168-169.

4. Hammond, P., Davenport, J. C., Fitzpatrick, F. J., Randell, D. A. and de Mattos, M. G. (1993) The RaPiD project: knowledge based design of dental prostheses. in Knowledge at Work in Universities. (ed Atwell, E. S.), Leeds University Press, pp. 70-81.

5. Basker, R. M., Harrison, A., Davenport, J. C. and Marshall, J. L. (1988) Partial denture design in general dental practice - 10 years on. British Dental Journal 165, pp. 245-249.

6. Pollard, D. and Davenport, J. C. (1994) An evaluation of training general dental practitioners in partial denture design via computer-assisted learning. British Dental Journal 177, pp. 405-409.

7. Davenport, J. C., Basker, R. M., Heath, J. R. and Ralph, J. P. (1988) A colour atlas of removable partial dentures. Wolfe Medical Publications Ltd, London.

8. Hammond, P., Davenport, J. C. and Fitzpatrick, F. J. (1993) Logic-based integrity constraints and the design of dental prostheses. Artificial Intelligence in Medicine 5, pp. 431-466. 
9. Johnson, L. and Johnson, N. E. (1987) Knowledge elicitation involving teachback interviewing, in Knowledge Elicitation for Expert Systems: A Practical Handbook. (ed A. Kidd), Plenum Press, New York.

10. Burton, A. M., Shadbolt, N. R., Rugg, G. and Hedgecock, A. P. (1990) The efficacy of knowledge elicitation techniques: a comparison across domains and levels of expertise. Knowledge Acquisition 2, pp. 167-178.

11. Grabowski, M., Massey, A. P., and Wallace, W. A. (1992) Focus groups as a group knowledge acquisition technique. Knowledge Acquisition 4, pp. 401-25.

12. Puerta, A. R., Egar, J. W., Tu, S. W. and Musen, M. A. (1992) A multiplemethod knowledge acquisition shell for the automatic generation of knowledgeacquisition tools. Knowledge Acquisition 4, pp. 171-196. 